\title{
Prescription Pattern of Chronic Kidney Disease Patients undergoing Hemodialysis in Tertiary and Private Hospital
}

\author{
Janet Mary Oommen', Dhanisha P. Nerurkar', Manjusha Sajith',", Sunil Jawale ${ }^{2}$, Shrinivas Ambike ${ }^{3}$ \\ 'Department of Clinical Pharmacy, Bharati Vidyapeeth Deemed to be University, Poona College of Pharmacy, Pune, Maharashtra, INDIA. \\ 2Department of Nephrology, Bharati Hospital and Research Centre, Pune, Maharashtra, INDIA. \\ 32Department of Nephrology, Jehangir Hospital, Pune, Maharashtra, INDIA.
}

\begin{abstract}
Objective: To evaluate the prescription pattern of chronic kidney disease patients undergoing haemodialysis in both hospitals. Methods: The study was carried out in a tertiary care and a private hospital for a period of nine months. Chronic kidney disease patients on maintenance haemodialysis for at least one month were included. Details like sociodemographic and clinical characteristics, past medication history, comorbidities and current medications were noted in self-pre-designed Patient Proforma. Mean \pm standard deviation and percentages and relevant statistical tests like Chisquare test was used. Results: Majority of the patients belonged to middle socio-economic class in private hospital and lower-middle class in tertiary hospital. Maximum were unemployed $(50.60 \%, 36 \%)$, married $(90.36 \%$, $88 \%)$ and had high school qualification $(62.65 \%, 45.33 \%)$. About 78 (93.97\%) patients were covered with insurance/health scheme in tertiary hospital and $39(52 \%)$ in private hospital. Hypertension was found to be the leading cause in tertiary and private hospital. Calcium channel blockers $(77.10 \%, 53.30 \%)$ were highly prescribed in both the hospitals. Erythropoi-
\end{abstract}

etin $(69.80 \%)$, calcium acetate $(21.70 \%$ ) and anti-diabetics (Insulin $10.84 \%)$ in tertiary hospital, whereas newer and costlier drugs like Darbepoetin, Iron preparations and Lanthanum carbonate were prescribed in private hospital. Conclusion: Socioeconomic status led to variation in prescription patterns among both hospitals as newer and costlier drugs like Lanthanum carbonate, Sevelamer and Darbepoetin were prescribed only in private hospital. Key words: Chronic kidney disease, Hemodialysis, Polypharmacy, Prescription pattern, Socioeconomic status.

Correspondence

Mrs. Manjusha Sajith, Department of Clinical Pharmacy, Bharati Vidyapeeth Deemed to be University Poona College of Pharmacy, Pune- 411043, Maharashtra, INDIA.

Phone: +919730080959

Email: manjusaji1@yahoo.com

DOI: 10.5530/jyp.2019.11.42

\section{INTRODUCTION}

Chronic Kidney Disease (CKD) is now recognized as a major medical problem worldwide. ${ }^{1}$ The Global Burden of Disease (GBD) study 2015 ranked chronic kidney disease as $17^{\text {th }}$ among the cause of deaths globally (Age-standardized annual death rate of 19.2 deaths per 100,000 population). ${ }^{2}$ Although the exact incidence and prevalence rates are not available, it is estimated that one out of 10,000 people suffer from CKD in India and around 100 thousand new patients develop End Stage Renal Disease (ESRD) in India annually. ${ }^{3}$ Chronic hemodialysis patients have numerous complications such as fluid retention, increase in potassium levels, low haemoglobin levels, weak bones requiring pharmacologic therapy. Multiple medications are essentially required to control comorbid conditions like hypertension, diabetes mellitus and cardiovascular diseases. It can increase the cost of treatment and also pose a challenge for the treatment of patients with CKD. Non-compliance with drug regimens may increase the risk of severe complications and represents a potential problem in hemodialysis patients who are on multiple medicines. There are some known predictors for multiple medications, i.e. age, female gender, low educational status. ${ }^{4}$ Lower socioeconomic status is linked to lower health education. ${ }^{5}$ The difference in the socioeconomic status may lead to variability in the prescription pattern among CKD patients.

Thus, the purpose of this study was to obtain information about the prescription pattern in CKD patients undergoing hemodialysis and observe the variations among both the hospitals.

\section{MATERIALS AND METHODS}

Study site selected was a tertiary hospital and a private hospital for a period of nine months from September 2017 to May 2018. Patients having CKD and on maintenance haemodialysis for a minimum of 1 month were included in our study. Exclusion criteria included pregnant women on dialysis, age less than 18 years, patients who are being dialyzed for Acute Kidney Injury and patients who are being evaluated for renal transplantation. Ethical approval was obtained for the study from the institutional ethics committee. The details like sociodemographic and clinical characteristics, past medication history, comorbidities and current medications (Number of medications, dose regimen and frequency) were noted in the self-pre-designed Patient Proforma. Mean \pm standard deviation and percentages was used for summarizing the data. Other relevant statistical tests Chi-square test was used for quantitative data and comparison of proportions. The $P$ value $<0.05$ was considered as statistical significant.

\section{RESULTS}

The highest number of patients undergoing hemodialysis in the both the hospitals were found to be males $(73.49 \%, 60 \%)$. The mean age in the patients undergoing hemodialysis in both the hospitals was $49.53 \pm$ 15.09 years and $51.52 \pm 14.53$ years. Majority of the patients in the private hospital were from the middle socio-economic class (80\%) whereas, in tertiary hospital subjects belonged to lower-middle socioeconomic class $(100 \%)$ which was found to be statistically significant $(P=<0.001)$. The mean duration of dialysis was $2.71 \pm 2.36$ in tertiary and $3.21 \pm 2.85$ 
in private hospital. The frequency of twice weekly dialysis was highest in tertiary hospital whereas thrice weekly in private hospital (Table 1). Hypertension $(100 \%, 89.06 \%)$ was the commonest comorbidity followed by diabetes plus hypertension $(35 \%, 31.25 \%)$ in both the hospitals (Table 2).

The total number of medications prescribed in 158 patients was 1262 out of which 623 were from tertiary hospital and 639 from private hospital. The average numbers of drugs per prescription from tertiary and private hospital were $7.51 \pm 2.09$ and $8.52 \pm 1.35$ respectively. Polypharmacy (use of $\geq 5$ medications) was observed in $86.75 \%$ patients in tertiary hospital and $97.33 \%$ patients in private hospital. Anti-coagulants were given to all patients during hemodialysis.

The most prescribed drugs in tertiary hospital were anti-hypertensive drugs (93.97\%), hematopoietic agents (72.28\%), Vitamins and mineral supplements $(65.06 \%)$ and the least prescribed were anti-arrhythmic drugs (1.21\%) and anti-thyroid drugs (1.21\%). In private hospital, hematopoietic agents (97.33\%), Vitamins and mineral supplements (94.66\%) and phosphate binders (93.33\%) were highly prescribed and least prescribed drugs were statins (2.66\%) (Figure 1).

Out of the anti-hypertensive drugs, calcium channel blockers were highly prescribed for the treatment of hypertension in both the hospitals (77.10\%, 53.30\%) (Figure 2). Among the Calcium Channel Blockers (CCBs), Amlodipine was most commonly prescribed both in tertiary $(65.60 \%)$ and in private $(92.50 \%)$ hospital. Other CCBs prescribed were Nifedipine, Clinidipine and Benidipine. Metoprolol was the commonest beta blocker prescribed for hypertension in tertiary and private hospital (91.6\% and $100 \%$ respectively). Hematopoietic agents used in tertiary and private hospital were Erythropoeitin/Epoetin alfa (69.87\% and $68.00 \%$ ) and iron preparations $(49.40 \%$ and $92.00 \%)$ respectively in parenteral forms. Iron preparations in tertiary hospital included iron sucrose $48.19 \%$ (Parenteral), ferrous sulphate $1.20 \%$ (Parenteral) and ferric citrate $1.20 \%$ (Oral) whereas in private hospital iron preparations include iron sucrose $92.00 \%$ (Parenteral) and ferric carboxymaltose $2.66 \%$ (Parenteral). The oral hypoglycemic drugs prescribed were $3.61 \%$ and $8 \%$ patients in tertiary and private hospital respectively (Figure 3). Insulin was slightly prescribed more in tertiary hospital $10.84 \%(n=9)$ than private hospital $8.00 \%(n=6)$. Among anti-secretory drugs, Proton Pump Inhibitors (PPIs) were more widely used than $\mathrm{H}_{2}$ blockers and antiemetics in both hospitals. The most commonly used PPIs were pantoprazole $84.60 \%(n=11)$ in tertiary and $95.20 \%(n=20)$ in private hospital (Figure 4). Phosphate binders were prescribed in the form of calcium and calcium free agents. Calcium Acetate was maximally prescribed in private hospital $22(29.33 \%)$ than in a tertiary setting 18 (21.68\%) (Figure 5). Lanthanum carbonate 58 (77.33\%) and Sevelamer 6 (8.00\%) was only prescribed in private hospital.

\section{DISCUSSION}

The majority of the patients undergoing hemodialysis were males which showed a consistent pattern when compared to Sanjay K. Agarwal et al. ${ }^{6}$ Manfred Hecking et al. ${ }^{7}$ and Lakshminarayan et al. ${ }^{8}$ who reported similar results. Males demonstrated higher risk factors such as overweightness, having greater waist circumference and an elevated blood pressure which makes them more vulnerable to develop chronic kidney disease over a period of time. ${ }^{9}$ On the contrary Lori L. Pounds et al. and Idan Goldberg et al. in their report stated that the prevalence of CKD tends to be higher in women, whereas the progression of disease is more severe in men. ${ }^{9,10}$ The mean age reported in tertiary care hospital and private hospital were found to be similar. Bernard Canaud et al. in his study stated that elderly patients account for an increased fraction of patients on renal replacement therapy worldwide due to ageing and underlying comorbidities $(25 \%-30 \%)^{11}$ thus justifying the higher number of our study subject belonging to the age group of 60 years and above $(30.12 \%$,

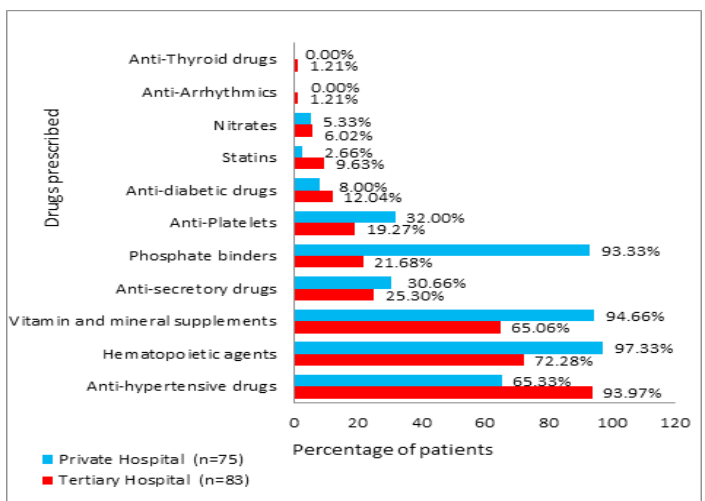

Figure 1: Categories of Drugs Prescribed in CKD Patients Undergoing Hemodialysis.

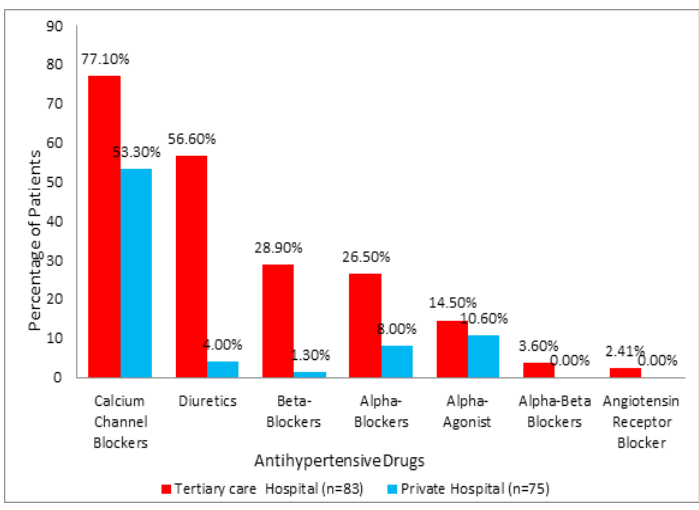

Figure 2: Anti-hypertensive Drugs Prescribed in CKD Patients Undergoing Hemodialysis.

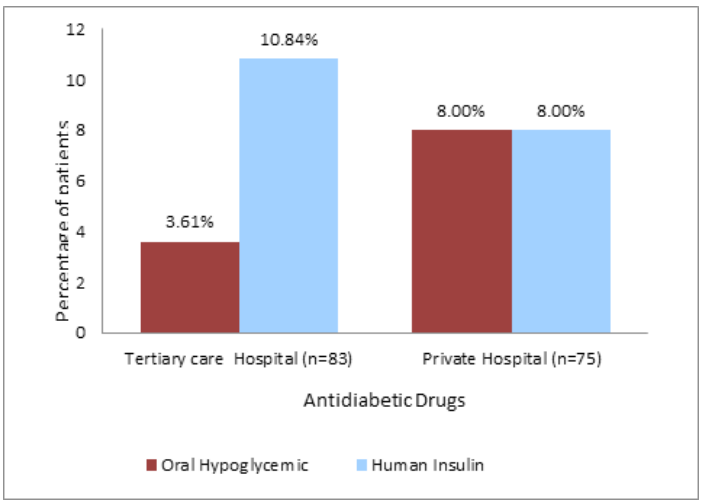

Figure 3: Anti-Diabetic Drugs Prescribed in CKD Patients Undergoing Hemodialysis.

$34.67 \%)$. In India hemodialysis is more preferred and is largely influenced by socioeconomic status and limited insurance schemes. ${ }^{12}$ In this study patients in private hospital were on thrice weekly hemodialysis, whereas in the tertiary care hospital they were on twice weekly hemodialysis majorly considering that a greater number of them belonged to the lower-middle socioeconomic class, were unemployed and economically depended on their family or insurance coverage for dialysis and drug expenditure. Duration of dialysis was similar in both the hospitals. Similar results were published by Brian Bieber et al. which manifested higher number of patients dependent on twice weekly hemodialysis. ${ }^{13}$ 


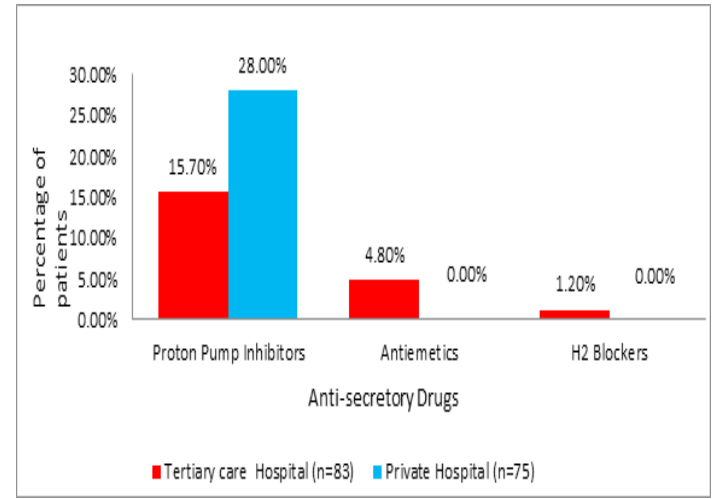

Figure 4: Prescription Pattern of Anti-Secretory Drugs in CKD Patients Undergoing Hemodialysis.

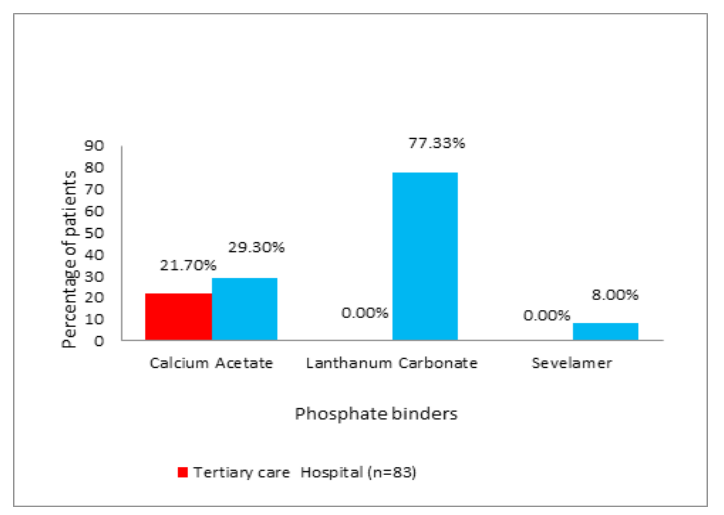

Figure 5: Phosphate Binders Prescribed in CKD Patients Undergoing Hemodialysis.

Hypertension can be a cause or a consequence of chronic kidney disease and has been reported to occur in $85 \%$ to $95 \%$ of patients with chronic kidney disease (Stages 3-5) in the United States. ${ }^{14}$ In present study, the medications were prescribed based on the comorbid conditions and complications involved during the patient treatment. Multiple medications are an unavoidable predicament faced during the management of CKD patients due to the prevalence of co-existing illnesses. In present study, polypharmacy (use of $\geq 5$ medications) was observed in $86.74 \%$ patients in tertiary hospital and $97.33 \%$ patients in private hospital. In a study by Chiu Y et al. the daily pill burden in the patients were very high and almost one-half of the patients were prescribed to take over 20 pills daily. ${ }^{15}$ The average number of drugs prescribed in the present study was almost similar in both the hospitals. Anti-coagulants were given during hemodialysis to all patients to prevent coagulation of blood during hemodialysis.

Hypertension is the most common comorbidity associated with Chronic Kidney Disease (CKD) followed by diabetes. In this study, the anti-hypertensive drugs were majorly prescribed in tertiary hospital whereas hematopoietic agents were maximally prescribed in private hospital. The most commonly used drugs for the treatment of hypertension were calcium channel blockers, followed by diuretics, beta blockers and alpha blockers in tertiary hospital whereas calcium channel blockers followed by alpha agonist, alpha blocker and diuretics were used in private hospital. All the antihypertensive drugs were prescribed in oral dosage form. Similar results were seen in studies conducted by Al Ramahi et al. Devi DP et al. and Bailie GR et al. ${ }^{16-18}$ Among the Calcium Channel Blockers (CCBs), Amlodipine was most commonly prescribed to reduce blood pressure for dialysis patients. Other CCBs prescribed were Nifedipine, Clinidipine and Benidipine in tertiary hospital whereas only Clinidipine and Nifedipine were given in private hospital. Metoprolol was the commonest beta blocker prescribed for hypertension in both tertiary and private hospital in this study. Other drugs used from this class included Nebivolol.

Diuretics were used commonly in about more than $50 \%$ of the patients especially loop diuretics like Furosemide (74.5\%) in tertiary hospital which is consistent with the studies conducted by Al Ramahi et al. Ahlawat $\mathrm{R}$ et al. ${ }^{16-19}$ Other classes of cardiovascular drugs prescribed were cardiac glycosides, anti-anginals, cholesterol lowering agents, antiarrhythmics. Cholesterol lowering agents like statins namely atorvastatin was prescribed more in tertiary than private hospital. In a study by $\mathrm{Al}-$ Ramahi et al. ${ }^{16}$ around $47 \%$ of the patients were using serum lipid reducing agents mostly Lovastatin.

Anemia is the most common complication due to reduced erythropoietin secretion. In this study, hematopoietic agents utilized in tertiary and private hospital were Erythropoietin/epoetin alfa (EPO) and iron preparations respectively which were found to be higher than a study conducted by Ahlawat $\mathrm{R}$ et al. ${ }^{19}$ and in a study by $\mathrm{Al}$ Ramahi et al. ${ }^{16}$ where erythropoietin was under prescribed and higher frequency of blood transfusion were compounded. Iron preparations were highly prescribed in private hospital than in tertiary hospital. Darbepoetin (DPO) was mostly prescribed in private hospital than tertiary hospital. As the maximum number of patients in tertiary hospital belonged to either lower/middle socioeconomic class, Erythropoietin was maximally given in both the hospitals. However, Santra $\mathrm{S}$ et al. and others, in their study reported that hematopoietic agents like Erythropoietin and Darbepoetin were underused due to low patient compliance and high cost. ${ }^{20}$ The prescription of water-soluble vitamin supplements is a regular practice in many dialysis units. Vitamins and minerals were prescribed maximally in private hospital. Folic acid was only utilized in private hospital (54.7\%). Vitamin B complex was prescribed in both the hospitals. A recent meta-analysis by Wang $L$ et al. included observational and randomized studies of vitamin D supplementation and concluded that on the basis of limited evidence, vitamin D supplements when given at moderate to high doses may reduce cardiovascular risk. ${ }^{21}$ Vitamin $\mathrm{D}$ analogues namely calcitriol and alfacalcidol were maximally prescribed in private hospital and minimally prescribed in tertiary hospital. Combination of calcium carbonate with vitamin $\mathrm{D}_{3}$ and vitamin $\mathrm{B}$ complex with iron was given only in tertiary hospital. It was found that not many could afford the higher cost of vitamin D analogues, so a combination therapy with calcium was given to patients in tertiary hospital.

Hyperphosphatemia is a frequently seen complication in patients with End Stage Renal Disease (ESRD). Phosphate binders are prescribed to CKD patients in the form of calcium and calcium free agents. In the present study, calcium acetate was maximally prescribed as a phosphate binder in both the hospitals. One research also stated that calcium acetate should be considered the calcium-based binder of choice in the management of uremic hyperphosphatemia. A study by Galani $\mathrm{V}$ et al. suggested that calcium acetate can be used for patients of lower socioeconomic status with hyperphosphatemia and hypocalcemia similar to present study. ${ }^{22}$ The high cost of treatment of Sevelamer hydrochloride and Lanthanum carbonate makes it less affordable for a good proportion of the Indian population. Calcium supplements are more affordable because of low cost when compared to Sevelamer. In the present study, Lanthanum carbonate and Sevelamer were only prescribed in private hospital. The high cost of Sevelamer hydrochloride can limit its use for the poor socioeconomic population therefore only prescribed in private hospital. Hyperglycemia is a fundamental cause of vascular target organ complications, including Diabetic Kidney Disease (DKD) and there is a 
Table 1: Sociodemographic and clinical characteristics of CKD patients undergoing hemodialysis.

\begin{tabular}{|c|c|c|c|}
\hline \multirow[b]{2}{*}{ Characteristics } & \multicolumn{2}{|c|}{ Number of patients (\%) } & \multirow[b]{2}{*}{$\begin{array}{c}\text { Total } \\
(n=158)\end{array}$} \\
\hline & Tertiary Hospital $(n=83)$ & Private Hospital $(n=75)$ & \\
\hline \multicolumn{4}{|c|}{ Gender } \\
\hline Male & $61(73.49)$ & $45(60)$ & $106(67.09)$ \\
\hline Female & $22(26.51)$ & $30(40)$ & $52(32.91)$ \\
\hline Mean Age (years) & $49.53 \pm 15.09$ & $51.52 \pm 14.53$ & \\
\hline \multicolumn{4}{|c|}{ Socioeconomic Class(based on Monthly Income) } \\
\hline Lower & $30(36.14)$ & $5(6.67)$ & $35(22.15)$ \\
\hline Lower middle & $7(8.43)$ & $8(10.67)$ & $15(9.49)$ \\
\hline Middle & $46(55.42)$ & $60(80)$ & $106(67.09)$ \\
\hline Upper middle & $0(0)$ & $1(1.33)$ & $1(0.63)$ \\
\hline Higher & $0(0)$ & $1(1.33)$ & $1(0.63)$ \\
\hline $\begin{array}{c}\text { Mean Duration of } \\
\text { dialysis (years) }\end{array}$ & $2.74 \pm 2.36$ & $3.21 \pm 2.85$ & \\
\hline \multicolumn{4}{|l|}{$\begin{array}{l}\text { Frequency of dialysis } \\
\text { session per week }\end{array}$} \\
\hline One time/ week & $6(7.23)$ & $1(1.33)$ & $7(4.43)$ \\
\hline Two times/ week & $65(78.31)$ & $36(48)$ & $101(63.92)$ \\
\hline Three times/ week & $12(14.46)$ & $38(50.67)$ & $50(31.65)$ \\
\hline
\end{tabular}

Table 2: Comorbidities of CKD Patients Undergoing Hemodialysis.

\begin{tabular}{|c|c|c|c|c|}
\hline \multicolumn{3}{|c|}{ Co-morbid Conditions } & \multirow{3}{*}{$\begin{array}{c}\text { Chi- square } \\
0.909\end{array}$} & \multirow{3}{*}{$\begin{array}{l}\text { P value } \\
0.340^{*}\end{array}$} \\
\hline Present & 80 & 64 & & \\
\hline Absent & 3 & 6 & & \\
\hline Hypertension & $\begin{array}{c}46 \\
(57.5)\end{array}$ & $35(54.69)$ & \multicolumn{2}{|c|}{$81(56.25)$} \\
\hline Diabetes+ Hypertension & $28(35)$ & $20(31.25)$ & \multicolumn{2}{|c|}{$48(33.33)$} \\
\hline Diabetes Mellitus & $0(0)$ & $5(7.81)$ & \multicolumn{2}{|c|}{$5(3.47)$} \\
\hline $\begin{array}{l}\text { Hypothyroidism/ } \\
\text { Hyperthyroidism }\end{array}$ & $0(0)$ & $2(3.13)$ & \multicolumn{2}{|c|}{$2(1.39)$} \\
\hline $\begin{array}{l}\text { Hypertension + } \\
\text { Hypothyroidism }\end{array}$ & $3(3.75)$ & $0(0)$ & \multicolumn{2}{|c|}{$3(2.08)$} \\
\hline $\begin{array}{c}\text { Hypertension }+ \text { Diabetes }+ \\
\text { Coronary artery disease }+ \\
\text { Hypothyroidism }\end{array}$ & $1(1.25)$ & $0(0)$ & \multicolumn{2}{|c|}{$1(0.69)$} \\
\hline $\begin{array}{l}\text { Hypertension }+ \text { Diabetes+ } \\
\text { Coronary artery disease }\end{array}$ & $1(1.25)$ & $0(0)$ & \multicolumn{2}{|c|}{$1(0.69)$} \\
\hline $\begin{array}{l}\text { Hypertension + Coronary } \\
\text { artery disease }\end{array}$ & $1(1.25)$ & $1(1.56)$ & \multicolumn{2}{|c|}{$2(1.39)$} \\
\hline $\begin{array}{c}\text { Hypertension + Diabetes + } \\
\text { COPD }\end{array}$ & $0(0)$ & $1(1.56)$ & \multicolumn{2}{|c|}{$1(0.69)$} \\
\hline
\end{tabular}

Chi-square test.

${ }^{*} \mathrm{P}<0.05$ is considered to be statistically significant.

greater need for stricter glycemic control in diabetic patients undergoing dialysis to prevent hypoglycemic episodes. Insulin was prescribed more in both hospitals as compared to oral hypoglycemic drugs which was consistent with a study carried out by Zaman Huri H. et al. where more than a half of diabetic patients with CKD were prescribed with insulin for their glycemic control. ${ }^{23}$ In this study, Meglitinides and DDP-4 inhibitors were the class of drugs preferred in tertiary hospital as opposed to a study where no monotherapy involving DPP-4 inhibitors were observed in the study population whereby they were prescribed other anti-diabetic combinations. Regular insulin and Isophane insulin were equally prescribed in both the hospitals whereas combinations of Insulin Neutral Protamine Hagedorn (NPH) (50\%) + Insulin Regular (50\%) and Human Insulin (30\%) + Human Isophane Insulin (70\%) were preferred in tertiary hospital.

Among anti-secretory drugs, Proton Pump Inhibitors (PPIs) were more widely used than $\mathrm{H}_{2}$ blockers and antiemetics in both hospitals. The most commonly used PPIs were Pantoprazole $84.6 \%(n=11)$ in tertiary and $95.2 \%(n=20)$ in private hospital. Apart from Pantoprazole, Rabeprazole was prescribed in tertiary hospital whereas Esomeprazole was prescribed in private hospital.

\section{CONCLUSION}

In the tertiary hospital, majority of the patients belonged to lowermiddle socioeconomic class and were covered under insurance/ health scheme. Hypertension was the most common cause of CKD and also the highest co-morbidity reported. Presence of multiple comorbidities led to polypharmacy in both the hospitals. Newer and costlier drugs like Lanthanum carbonate, Sevelamer and Darbepoetin were more prescribed in private hospital than tertiary hospital where Calcium acetate and Erythropoietin was prescribed. Hematopoietic agents, vitamin and mineral supplements were majorly prescribed in both the hospitals, which were followed by prescription of anti-hypertensives in tertiary hospital and phosphate binders in the private hospital. Prescribing pattern of private hospital, where newer and costlier drugs were prescribed, varied from tertiary hospital based on the socioeconomic status of the patients.

\section{ACKNOWLEDGEMENT}

The authors thank all the staff including residents, doctors and nurses of Bharati Hospital and Research Centre, Bharati Vidyapeeth Deemed Uni- 
versity Pune and Jehangir Hospital, Pune, for helping us in the completion of this study.

\section{CONFLICT OF INTEREST}

The authors declare no conflict of interest.

\section{REFERENCES}

1. Jha V, Garcia-Garcia G, Iseki K, Li Z, Naicker S, Plattner B, et al. Chronic kidney disease: Global dimension and perspectives. Lancet. 2013;382(9888):260-2.

2. Wang H, Naghavi M, Allen C, Barber R, Bhutta Z, Carter A, et al. Global, regional and national life expectancy, all-cause mortality and cause-specific mortality for 249 causes of death, 1980-2015: A systematic analysis for the Global Burden of Disease Study 2015. Lancet. 2019.

3. Acharya L, Attur R, Rama M, Viswanathan G, Reddy P, Raghavan S. Assessment of drug-drug interactions among renal failure patients of nephrology ward in a south Indian tertiary care hospital. Indian J Pharm Sci. 2012;74(1):63-8.

4. Strehblow C, Smeikal M, Fasching P. Polypharmacy and excessive polypharmacy in octogenarians and older acutely hospitalized patients. Wien Klin Wochenschr. 2014:126(7-8):195-200.

5. Fraser S, Roderick P, May C, Mclntyre N, Mclntyre C, Fluck R, et al. The burden of comorbidity in people with chronic kidney disease stage 3: A cohort study. BMC Nephrol. 2015;16(1):193.

6. Agarwal SK, Dash SC, Irshad M, Raju S, Singh R, Pandey RM. Prevalence of chronic renal failure in adults in Delhi, India. Nephrol Dial Transplant. 2005;20(8):1638-2.

7. Hecking M, Bieber B, Ethier J, Kautzky-Willer A, Sunder-Plassmann G, Säemann M, et al. Sex-Specific Differences in Hemodialysis Prevalence and Practices and the Male-to-Female Mortality Rate: The Dialysis Outcomes and Practice Patterns Study (DOPPS). PLoS Med. 2014;11(10):e1001750

8. Lakshminarayana G, Sheetal L, Mathew A, Rajesh R, Kurian G, Unni V. Hemodialysis outcomes and practice patterns in end-stage renal disease: Experience from a Tertiary Care Hospital in Kerala. Indian J Nephrol. 2017;27(1):51-7.

9. Pounds L, Teodorescu V. Chronic kidney disease and dialysis access in women. J Vasc Surg. 2013;57(4):49-3.

10. Goldberg I, Krause I. The role of gender in chronic kidney disease. EMJ. 2016;1(2):58-4.

11. Canaud B, Tong L, Tentori F, Akiba T, Karaboyas A, Gillespie B, et al. Clinical practices and outcomes in elderly hemodialysis patients: results from the Dialysis Outcomes and Practice Patterns Study (DOPPS). Clin J Am Soc Nephrol. 2011;6(7):1651-2.

12. Mukherjee T, Devi G, Geetha S, Anchan N, Subbaiyan S. A comparison of prac- tice pattern and outcome of twice-weekly and thrice-weekly hemodialysis patients. Indian J Nephrol. 2017;27(3):185-9.

13. Bieber B, Qian J, Anand S, Yan Y, Chen N, Wang M, et al. Two-times weekly hemodialysis in China: Frequency, associated patient and treatment characteristics and Quality of Life in the China Dialysis Outcomes and Practice Patterns study. Nephrol Dial Transplant. 2013;29(9):1770-7.

14. Leticia BC. Chronic Kidney Disease and Hypertension: A Destructive Combination. Uspharmacist.com. 2019 [cited 26 October 2018]. Available from: https:// www. uspharmacist.com/article/chronic-kidney-disease-and-hypertension-adestructive-combination-35118

15. Chiu Y, Teitelbaum I, Misra M, DeLeon E, Adzize T, Mehrotra R, et al. Pill Burden, Adherence, Hyperphosphatemia and Quality of Life in Maintenance Dialysis Patients. Clin J Am Soc Nephrol. 2009;4(6):1089-6.

16. Al-Ramahi R. Medication prescribing patterns among chronic kidney disease patients in a hospital in Malaysia. Saudi J Kidney Dis Transpl. 2012;23(2):403-8.

17. Devi DP, George J. Diabetic nephropathy: Prescription trends in tertiary care. Indian J Pharm Sci. 2008;70(3):374-8.

18. Bailie GR, Eisele G, Liu L, Roys E, Kiser M, Finkelstein F, et al. Patterns of medication use in the RRI-CKD study: Focus on medications with cardiovascular effects. Nephrol Dial Transplant. 2005;20(6):1110-5.

19. Ahlawat R, D'cruz S. Drug Utilization Pattern in Chronic Kidney Disease Patients at a Tertiary Care Public Teaching Hospital: Evidence from a Cross- Sectional Study. J Pharma Care Health Sys. 2016;03(01)

20. Santra S, Agrawal D, Kumar S, Mishra S. A Study on the Drug Utilization Pattern in Patients with Chronic Kidney Disease with Emphasis on Antibiotics. J Integr Nephrol Androl. 2015;2(3):85-9.

21. Wang L, Manson JE, Song Y, Sesso HD. Systematic review: Vitamin D and calcium supplementation in prevention of cardiovascular events. Ann Intern Med. 2010;152(5):315-3.

22. Galani V, Prajapati V, Shah P. A comparative study of phosphate binders in patients with end stage kidney disease undergoing hemodialysis. Saudi J Kidney Dis Transpl. 2014;25(3):530-8.

23. Zaman HH, Lim L, Lim S. Glycemic control and antidiabetic drugs in type 2 diabetes mellitus patients with renal complications. Drug Des Devel Ther. 2015;9:4355.

Article History: Submission Date : 26-10-2018; Revised Date : 30-11-2018; Acceptance Date : 26-02-2019.

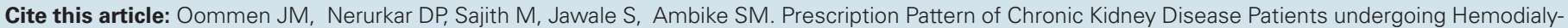
sis in Tertiary and Private Hospital. J Young Pharm. 2019;11(2):202-6. 